\title{
Scientific rationale underlying the use of nicotinamide in actinic keratosis
}

\author{
R. Di Caprio', A. Balato², N. Balato', S. Lembo³, G. Monfrecola1 \\ 1Department of Clinical Medicine and Surgery, University of Naples Federico II, Italy \\ 2Department of Advanced Biomedical Sciences, University of Naples Federico II, Italy \\ ${ }^{3}$ Department of Medicine, Surgery and Dentistry, Scuola Medica Salernitana, University of Salerno, Italy
}

\section{INTRODUCTION:}

Besides its known activity in the promotion and progression of non-melanoma skin cancers (NMSCs), cyclooxygenase (COX-2) plays also an important role in modulating inflammatory processes, representing an important link between chronic inflammation and carcinogenesis. Indeed, nicotinamide (NCT), the amide active form of Vitamin B3, has proven to have anti-inflammatory and cancer protection properties, being able to reduce actinic keratosis formation as well as basal- and squamous-cell cancer rate in NMSCs high-risk patients.

\section{AMM OF THE STUDY}

\section{to investigate whether NGT was able to modulate}

\section{UVB-medfated cox-2 expression in immortalized kerathooytes}

\section{MATERIALS AND METHODS:}

Immortalized human keratinocytes were pre-incubated with different doses of NCT $(0.1,2.5$ and $10 \mathrm{mM})$ for $2 \mathrm{~h}$ and irradiated in PBS with UVB 10 and $60 \mathrm{~mJ} / \mathrm{cm}^{2}$. Immediately after irradiation, PBS was removed and replaced with the culture medium containing NCT at the above indicated concentrations. COX-2 gene expression was assessed after 6 and $24 \mathrm{~h}$, whereas protein levels were measured in cell lysates $24 \mathrm{~h}$ after irradiation.

\section{RESULTS}

(a)

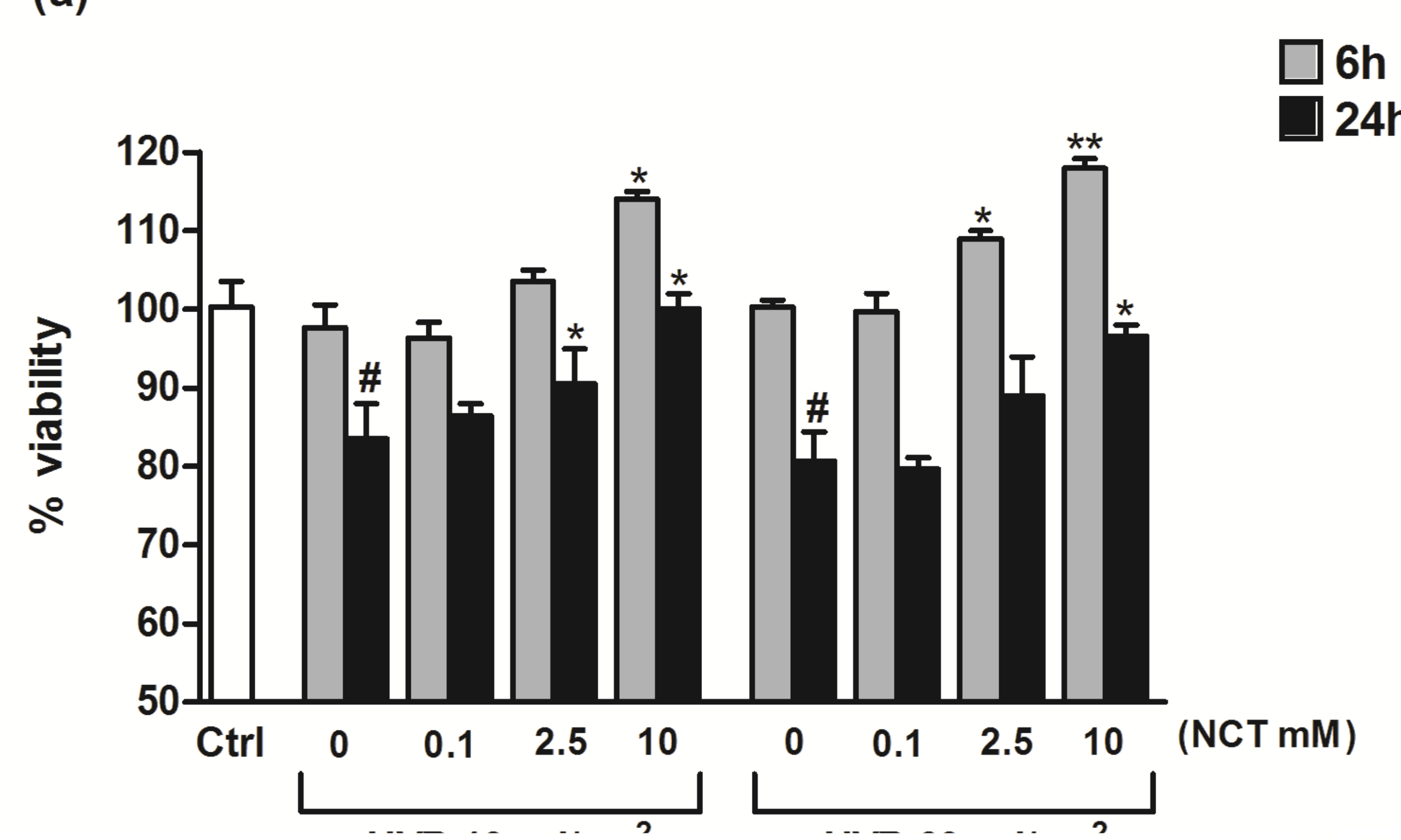

(b)

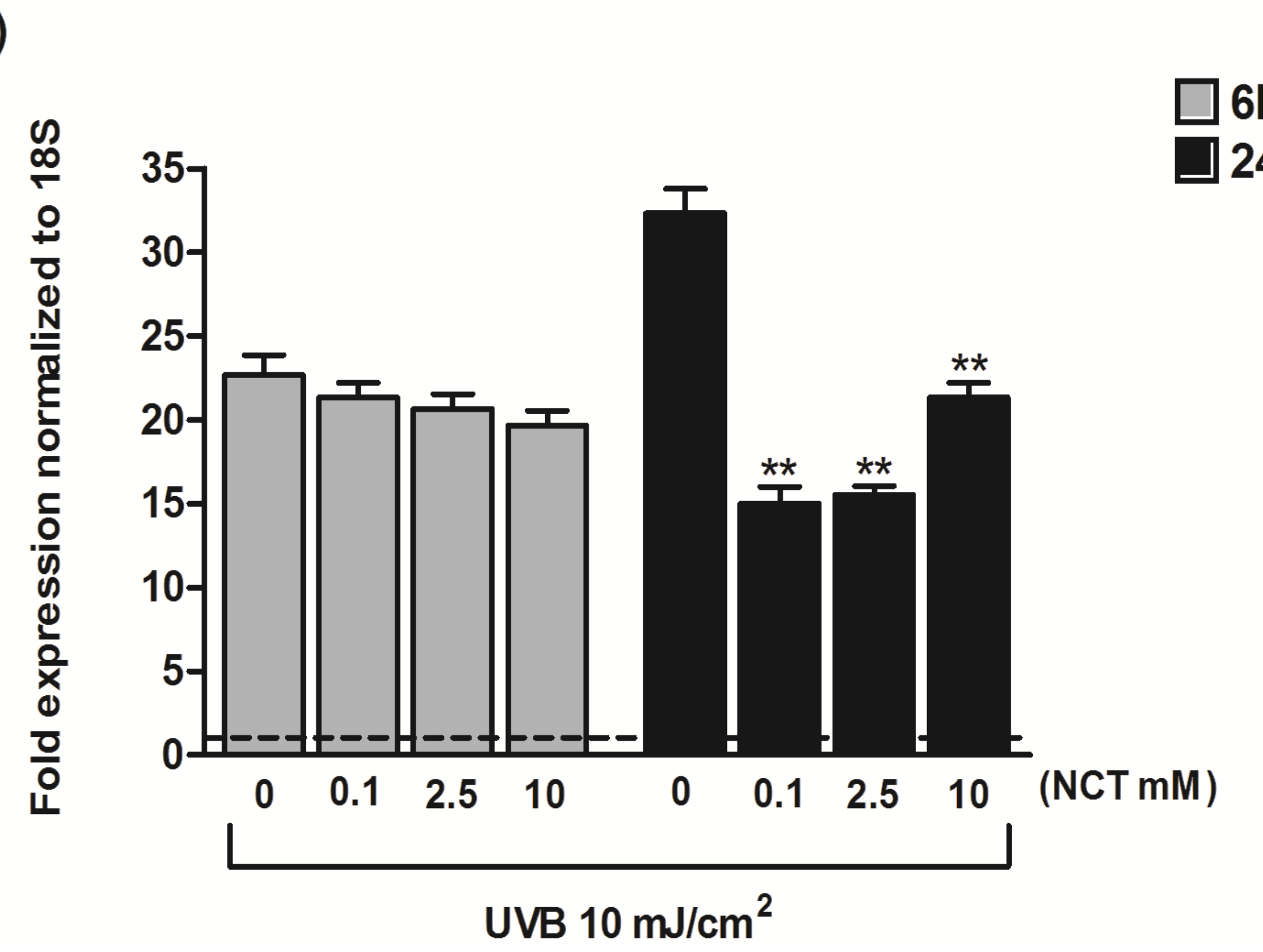

The selected in vitro doses of NCT of 0.1 and $2.5 \mathrm{mM}$ were calculated to approximately reproduce 10 times lower and 2 times higher doses of NCT plasma levels that can be obtained in humans after very high dose oral NCT. UVB dosages were chosen taken into account that they represent the right balance between cell viability (a) and a translational impact since mean UVB minimal erythema dose for a healthy population coming from our area (Southern Italy) is $75.3 \mathrm{~mJ} / \mathrm{cm}^{2}$

(c)

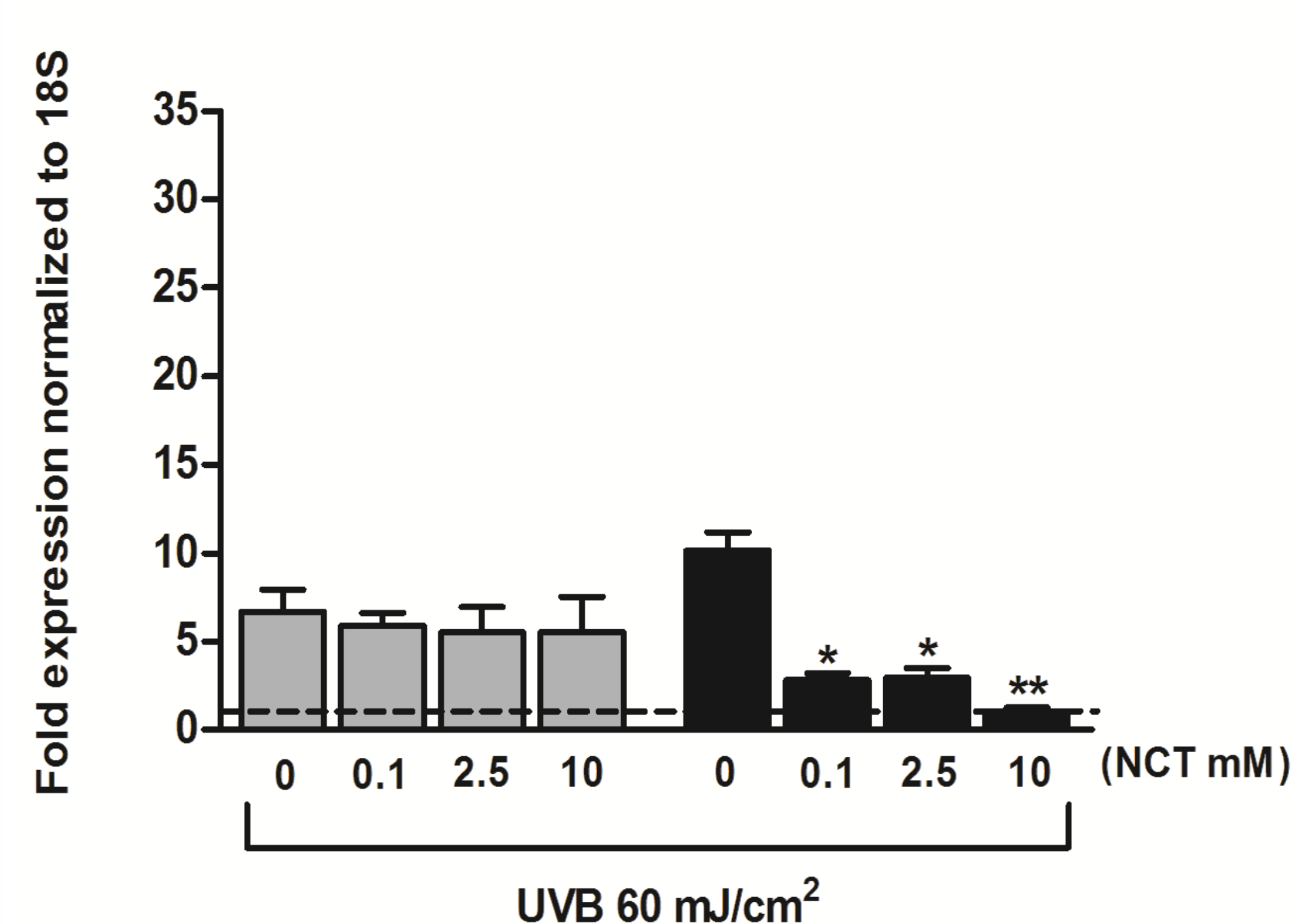

(d)

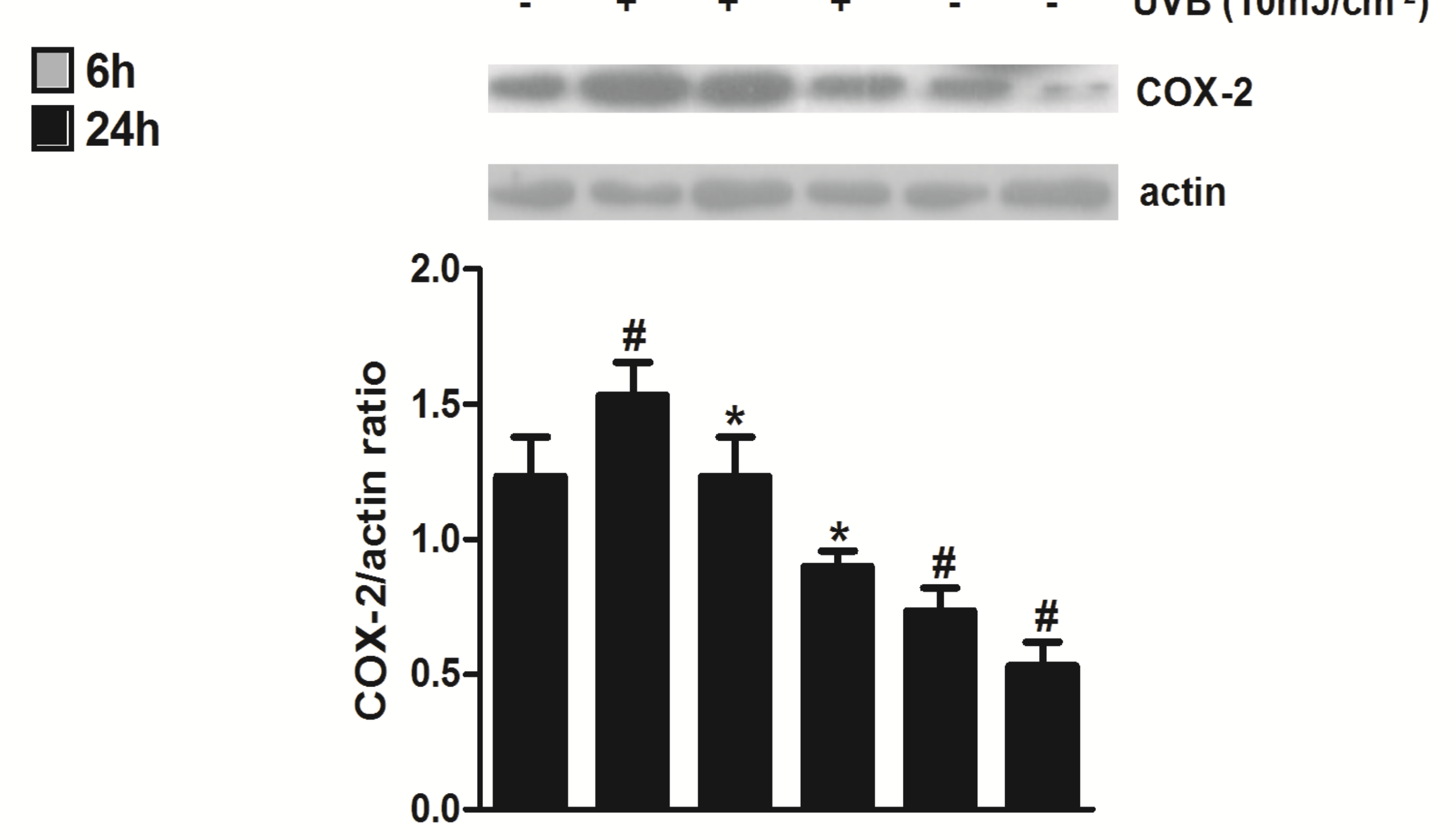

Pre-incubation for $2 \mathrm{~h}$ with NCT $0.1,2.5$ as well as $10 \mathrm{mM}$, strongly down-regulated COX-2 mRNA and protein $24 \mathrm{~h}$ after irradiation with UVB $10 \mathrm{~mJ} / \mathrm{cm}^{2}(\mathrm{~b}, \mathrm{~d})$. Irradiation with UVB $60 \mathrm{~mJ} / \mathrm{cm}^{2}$ resulted in a lower COX2 increase, nevertheless NCT was able to reduce its expression (c). Moreover, NCT treatment at high concentrations without UVB irradiation was able to decrease COX-2 (d).

\section{GONGLUSIONSE}

